\title{
Constructing Cut Free Sequent Systems with Context Restrictions Based on Classical or Intuitionistic Logic ${ }^{\star}$
}

\author{
Björn Lellmann ${ }^{1}$ and Dirk Pattinson ${ }^{2}$ \\ 1 Department of Computing, Imperial College London, UK \\ 2 Research School of Computer Science, The Australian National University
}

\begin{abstract}
We consider a general format for sequent rules for not necessarily normal modal logics based on classical or intuitionistic propositional logic and provide relatively simple local conditions ensuring cut elimination for such rule sets. The rule format encompasses e.g. rules for the boolean connectives and transitive modal logics such as $S 4$ or its constructive version. We also adapt the method of constructing suitable rule sets by saturation to the intuitionistic setting and provide a criterium for translating axioms for intuitionistic modal logics into sequent rules. Examples include constructive modal logics and conditional logic $\mathbb{V} \mathbb{A}$.
\end{abstract}

\section{Introduction}

It can hardly be disputed that cut elimination theorems are at the foundation of both theoretical investigation and practical implementation of automated reasoning techniques: the ensuing subformula property implies not only decidability of many logical systems, but also lies - mostly in the form of tableau methods - at the heart of the vast majority of implementations of various logics. Achieving cut elimination is usually a two stage process. First, a (sound and complete) set of sequent rules needs to be exhibited. Second, cut elimination is established. Both steps are equally laborious: finding the 'right' set of rules requires ingenuity and (syntactic) proofs of cut elimination rely on the judicious analysis of a large number of cases. Given the growth of logical systems of interest in particular in computer science, both generic methods with efficient tools for designing cut-free calculi, and meta-theorems that guarantee cut-elimination, decidability, and complexity bounds are therefore increasingly important.

This paper explores the method of cut elimination by saturation and extends previous work into two important directions. First, we can now also allow for the propositional base logic to be intuitionistic which allows us to treat a range of logics that have attracted interest in computer science [17/2]. Second, we generalise the approach to logics given by axioms of arbitrary modal rank. This is achieved by considering sequent rules with context restrictions where each premiss only propagates context formulae of a specific form. A prime example for this rule format are e.g. the rules of modal logic S4, where a premiss e.g. copies only boxed formulae on the left hand side. This extended rule format necessitates an extension of the previous characterisation of cut-free systems to deal with additional cases in the proof of cut elimination. In order to make full use of the extended rule format we investigate a method for translating axioms into

` Supported by EPSRC-Project EP/H016317/1.

K. Lodaya (Ed.): ICLA 2013, LNAI 7750, pp. 148-160, 2013.

(C) Springer-Verlag Berlin Heidelberg 2013 
rules which works uniformly for classical and intuitionistic logics. The rules so constructed are by construction sound and complete (in the presence of cut) and give rise to unlabelled sequent systems that are amenable to saturation under cuts between rules. In case the resulting rules fulfil our criteria for cut elimination and are also tractable they give rise to a generic ExPTIME decision algorithm for the logic. Our main contributions are the following: we formalise the notion of a rule with context restrictions (Definition 3), give a general criterion for cut elimination to obtain for a large class of modal logics extending classical or intuitionistic propositional logic (Theorem 16 ), and show how to construct sequent systems satisfying these requirements from axioms of a certain form (Section 3.2). We illustrate these techniques by reconstructing known cut-free sequent systems for constructive $S 4$, constructive $K$ and access control logic $C D D$, and also obtain a new cut-free calculus for Lewis' conditional logic $V A$. The techniques used are easily modified to treat e.g. minimal logic [9] or the $\{\wedge, \vee\}$-fragment of intuitionistic logic as base logics, but since we are not aware of modal logics based on either of these we restrict ourselves to the classical and intuitionistic cases.

Related Work: The method of cut elimination by saturation for extensions of classical logic with non-nested axioms was explored e.g. in [16 10]. The idea of contraction closed rule sets for first order and modal logics seems to have been formulated for the first time in [15 14], where also translations of axioms into rules of a labelled sequent system are given. Our rules with context restrictions are weaker versions of the rules with context relations from [3], which also allow the context formulae to change. While context relations are more general than context restrictions, apparently no syntactical criteria for cut elimination in such systems have been established yet. Our translations of axioms for intuitionistic modal logics into rules are motivated by the translations of (non-modal) axioms into structural rules for substructural logic in [6].

\section{Preliminaries}

Throughout, $\mathcal{V}$ denotes a denumerable set of propositional variables and $\Lambda$ is a set of connectives with associated arities. We write $\boldsymbol{p}$ for finite sequences of propositional variables. The set of $\Lambda$-formulae is defined by $\mathcal{F}(\Lambda) \ni A_{1}, \ldots, A_{n}::=p \mid \varnothing\left(A_{1}, \ldots, A_{n}\right)$ for $p \in \mathcal{V}$ and $\varnothing \in \Lambda$ with arity $n$. We write $\Lambda(S)=\left\{\varnothing\left(A_{1}, \ldots, A_{n}\right) \mid \varnothing \in\right.$ $\Lambda n$-ary, $\left.A_{1}, \ldots, A_{n} \in S\right\}$ for the set of formulae constructed from $S$ using a single connective in $\Lambda$. Uniform substitution of all propositional variables in a formula $A$ using a substitution $\sigma: \mathcal{V} \rightarrow \mathcal{F}(\Lambda)$ is denoted by $A \sigma$. The set $\mathcal{S}(F)$ of (symmetric) sequents over $F$ consists of tuples of multisets $\Gamma, \Delta$ of formulae in $F$, written $\Gamma \Rightarrow \Delta$. When dealing with extensions of intuitionistic propositional logic we consider asymmetric sequents, in which the right hand side $\Delta$ consists of at most one formula. The formulae in $\Gamma$ occur negatively in the sequent, those in $\Delta$ positively. The multiset union of two multisets $\Gamma$ and $\Delta$ is written $\Gamma, \Delta$ and we identify formulae with singleton multisets. Substitution extends to both multisets of formulae and sequents in the obvious way (perserving multiplicity), e.g. $\left(A_{1}, A_{2} \Rightarrow B\right) \sigma=A_{1} \sigma, A_{2} \sigma \Rightarrow B \sigma$. We use the systems $\mathrm{G} 2 c p$ and $\mathrm{G} 2 i p$ of [18] with axioms $\Gamma, A \Rightarrow \Delta, A$ (where $A$ ranges over the set of formulae) and the intuitionistic left implication rule $\frac{\Gamma \Rightarrow A \quad \Gamma, B \Rightarrow C}{\Gamma, A \rightarrow B \Rightarrow C}$ as basis for all 
systems that extend classical respectively intuitionistic propositional logic and write $\mathrm{G}$ resp. $\mathrm{G} i$ for these sets of rules. Our structural rules are

$\frac{\Gamma \Rightarrow \Delta}{\Sigma, \Gamma \Rightarrow \Delta, \Pi} \mathrm{W}, \frac{\Gamma, A, A \Rightarrow \Delta}{\Gamma, A \Rightarrow \Delta} \mathrm{ConL}, \frac{\Gamma \Rightarrow \Delta, A, A}{\Gamma \Rightarrow \Delta, A} \operatorname{ConR}, \frac{\Gamma \Rightarrow \Delta, A \quad A, \Sigma \Rightarrow \Pi}{\Gamma, \Sigma \Rightarrow \Delta, \Pi}$ Cut .

\section{Generic Cut Elimination and Construction of Cut-Free Systems}

We start our investigation with the observation that while standard sequent rules for the boolean connectives carry over the whole context to the premisses, in standard sequent systems for many modal logics such as $K$ or $S 4$ [19] either no or only modalised context formulae are propagated from conclusion to premisses. At the same time exactly one layer of modalities is added to the principal formulae. In order to fit these different formats into a unified framework we now generalise the notion of a shallow rule [10] using the notion of context restrictions, a weaker form of the context relations in [3].

Definition 1. If $F$ is a set of formulae, a context restriction $C$ over $F$ (or simply a restriction) is given by a tuple of sets of formulae in $F$, i.e. $C=\left\langle F_{1}, F_{2}\right\rangle$ with $F_{1}, F_{2} \subseteq$ $F$. We write $\mathfrak{C}(F)$ for the set of context restrictions over $F$. For a restriction $C=\left\langle F_{1}, F_{2}\right\rangle$ and a sequent $\Gamma \Rightarrow \Delta$ we write $(\Gamma \Rightarrow \Delta) \uparrow_{C}$ or $\Gamma \uparrow_{F_{1}} \Rightarrow \Delta \uparrow_{F_{2}}$ for the sequent consisting of the restriction of $\Gamma$ (resp. $\Delta$ ) to substitution instances of formulae $A$ with $A \in F_{1}$ (resp. $A \in F_{2}$ ) on the left (resp. right) hand side. An occurrence of a formula in a sequent $\Gamma \Rightarrow \Delta$ satisfies context restriction $C$ if it also occurs in $(\Gamma \Rightarrow \Delta) \uparrow_{C}$, and a sequent $\Gamma \Rightarrow \Delta$ satisfies $C$ if $(\Gamma \Rightarrow \Delta) \Upsilon_{C}=\Gamma \Rightarrow \Delta$. Finally, a context restriction $C^{\prime}$ satisfies $C$ if every sequent which satisfies $C^{\prime}$ also satisfies $C$.

Example 2. 1. The trivial restriction $C_{i d}:=\langle\{p\},\{p\}\rangle$ does not restrict a sequent at all, we always have $(\Gamma \Rightarrow \Delta) \uparrow c_{i d}=\Gamma \Rightarrow \Delta$.

2. The empty restriction $C_{\emptyset}:=\langle\emptyset, \emptyset\rangle$ deletes every formula in a sequent: $(\Gamma \Rightarrow$ 4) $\uparrow c_{\emptyset}=\Rightarrow$.

3. The restriction $C_{4 \square}:=\langle\{\square p\}, \emptyset\rangle$ deletes the right side of a sequent and restricts the left side to boxed formulae. E.g.: $(A, C \wedge D, \square(A \vee B) \Rightarrow \square D, B) \uparrow_{C_{4}}=\square(A \vee B) \Rightarrow$.

Definition 3. A rule with context restrictions (or simply a rule) is a tuple ( $\mathcal{P} ; \Sigma \Rightarrow \Pi$ ) where $\mathcal{P} \subseteq \mathcal{S}(\mathcal{V}) \times \mathfrak{C}(\mathcal{F})$ is the set of premisses with associated context restrictions, and $\Sigma \Rightarrow \Pi \in \mathcal{S}(\Lambda(\mathcal{V}))$ are the principal formulae, such that no variable occurs twice in the principal formulae and every variable occurs in the principal formulae if it occurs in at least one of the premisses. An instance of a rule $R$ is given by a substitution $\sigma: \mathcal{V} \rightarrow \mathcal{F}$ and a context $\Gamma \Rightarrow \Delta \in \mathcal{S}(\mathcal{F})$ and is written as

$$
\frac{\left\{\Gamma \uparrow_{F_{1}}, \Theta \sigma \Rightarrow \Delta \uparrow_{F_{2}}, \Upsilon \sigma \mid\left(\Theta \Rightarrow \Upsilon ;\left\langle F_{1}, F_{2}\right\rangle\right) \in \mathcal{P}\right\}}{\Gamma, \Sigma \sigma \Rightarrow \Delta, \Pi \sigma}
$$

Whenever we mention a set of rules we assume that it is closed under injective renaming of variables and for all $n$-ary $\nabla \in \Lambda$ and $\boldsymbol{p}=\left(p_{1}, \ldots, p_{n}\right)$ and $\boldsymbol{q}=\left(q_{1}, \ldots, q_{n}\right)$ includes the congruence rules $\left(\left\{\left(p_{i} \Rightarrow q_{i} ; \mathcal{C}_{\emptyset}\right) \mid i \leq n\right\} \cup\left\{\left(q_{i} \Rightarrow p_{i} ; \mathcal{C}_{\emptyset}\right) \mid i \leq n\right\} ; \diamond \boldsymbol{p} \Rightarrow \triangleright \boldsymbol{q}\right)$. 
Table 1. Some sequent rules as rules with context restrictions and in standard notation [18 19]

$$
\begin{array}{|ll}
\hline R_{K_{n}}:=\left(\left\{\left(p_{1}, \ldots, p_{n} \Rightarrow q ; C_{\emptyset}\right)\right\} ; \square p_{1}, \ldots, \square p_{n} \Rightarrow \square q\right) & \frac{A_{1}, \ldots, A_{n} \Rightarrow B}{\Gamma, \square A_{1}, \ldots, \square A_{n} \Rightarrow \Delta, \square B} \\
R_{T \square}:=\left(\left\{\left(p \Rightarrow ; C_{i d}\right)\right\} ; \square p \Rightarrow\right) & \frac{\Gamma, A \Rightarrow \Delta}{\Gamma, \square A \Rightarrow \Delta} \\
R_{4 \square}:=\left(\left\{\left(\Rightarrow p ; C_{4 \square}\right)\right\} ; \Rightarrow \square p\right) & \frac{\square \Sigma \Rightarrow A}{\Gamma, \square \Sigma \Rightarrow \Delta, \square A}
\end{array}
$$

Thus if a formula $A$ is in the left component of a restriction associated with a premiss of a rule, then in an instance of this rule the premiss carries over all substitution instances of $A$ from the left hand side of the context, and dually for the right hand side.

Example 4. The rules of $\mathrm{G}$ as well as the rules $\mathcal{R}_{K}:=\left\{R_{K_{n}} \mid n \geq 0\right\}$ of modal logic $K$ and $\mathcal{R}_{S 4}:=\left\{R_{T \square}, R_{4 \square}\right\}$ of modal logic $S 4$ from Table 1 are rules with context restrictions.

Definition 5. Let $\mathcal{R}$ be a set of rules and $S \subseteq \mathcal{S}(\mathcal{F})$ a set of sequents. We use the standard notion of derivations [18] and say that a sequent $\Gamma \Rightarrow \Delta$ is $\mathcal{R}$-derivable from $S$ if there is a derivation of $\Gamma \Rightarrow \Delta$ from $S$ using only instances of rules in $\mathcal{R}$. We then write $S \vdash_{\mathcal{R}} \Gamma \Rightarrow \Delta$. If we consider rules for asymmetric sequents we will indicate this by writing $\vdash_{\mathcal{R}}^{i}$, and we write $\vdash_{\mathcal{R}}^{[i]}$ if a result holds in both settings. Derivability from $\emptyset$ is denoted by $\vdash_{\mathcal{R}}^{[i]} \Gamma \Rightarrow \Delta$ and derivability in $\mathcal{R}_{1} \cup \mathcal{R}_{2}$ by $\vdash_{\mathcal{R}_{1} \mathcal{R}_{2}}^{[i]}$. We write $\mathcal{R}[$ CutCon] if a statement holds for $\mathcal{R}$ and extensions with Cut and / or Con.

Admissibility of Weakening is shown by a standard induction on the derivations:

Lemma 6. For every set $\mathcal{R}$ of rules and (asymmetric) sequent $\Gamma \Rightarrow \Delta$ we have ${ }_{\mathcal{R}}^{[i]}[\mathrm{CutCon}]$ $\Gamma \Rightarrow \Delta$ whenever $\vdash_{\mathcal{R} W[\text { CutCon }]}^{[i]} \Gamma \Rightarrow \Delta$.

\subsection{Cuts between Rules and Cut Elimination}

The main tool in the construction of cut free rule sets is the notion of cuts between rules from [1011] that we need to adapt to handle context restrictions. Cut between rules is a two-stage process: first we replace a pair of rules by the rule arising from performing a cut between the conclusions. In a second step we modify the premisses so that variables that no longer appear in the conclusion of the cut are eliminated.

Definition 7. If $\mathcal{P} \subseteq \mathcal{S}(\mathcal{V}) \times \mathfrak{C}(\mathcal{F})$ is a set of premisses with context restrictions, then for $p \in \mathcal{V}$ the $p$-elimination of $\mathcal{P}$ is the set

$$
\begin{aligned}
\mathcal{P} \ominus p:= & \left\{\left(\Gamma, \Sigma \Rightarrow \Delta, \Pi ; C_{1} \cup C_{2}\right) \mid\left(\Gamma \Rightarrow \Delta, p ; C_{1}\right) \in \mathcal{P},\left(p, \Sigma \Rightarrow \Pi ; C_{2}\right) \in \mathcal{P}\right\} \\
& \cup\{(\Gamma \Rightarrow \Delta ; C) \mid(\Gamma \Rightarrow \Delta ; C) \in \mathcal{P}, p \notin \Gamma, \Delta\},
\end{aligned}
$$

where for restrictions $C_{1}=\left\langle F_{1}, F_{2}\right\rangle$ and $C_{2}=\left\langle G_{1}, G_{2}\right\rangle$ we write $C_{1} \cup C_{2}$ for $\left\langle F_{1} \cup\right.$ $\left.G_{1}, F_{2} \cup G_{2}\right\rangle$. Iterated elimination of variables $\boldsymbol{p}=p_{1}, \ldots, p_{n}$ is denoted by $\mathcal{P} \ominus \boldsymbol{p}$. For rules $R=\left(\mathcal{P}_{R} ; \Gamma \Rightarrow \Delta, \odot \boldsymbol{p}\right)$ and $Q=\left(\mathcal{P}_{Q} ; \odot \boldsymbol{p}, \Sigma \Rightarrow \Pi\right)$ the cut between $R$ and $Q$ on $\odot \boldsymbol{p}$ is the rule $\operatorname{cut}(R, Q, \odot \boldsymbol{p}):=\left(\left(\mathcal{P}_{R} \cup \mathcal{P}_{Q}\right) \ominus \boldsymbol{p} ; \Gamma, \Sigma \Rightarrow \Delta, \Pi\right)$. A rule set $\mathcal{R}$ is principal-cut closed if it is closed under cuts between rules. 
Example 8. 1. The rule sets $\mathrm{G}[i]$ are principal-cut closed, since cuts between rules can be replaced by the identity rule $R_{i d}:=\left(\left\{\left(\Rightarrow ; C_{i d}\right)\right\} ; \Rightarrow\right)$.

2. The rule set $\mathcal{R}_{K}$ is principal-cut closed, since $\operatorname{cut}\left(R_{K_{n}}, R_{K_{m}}, \square q\right)=R_{K_{n+m-1}} \in \mathcal{R}_{K}$.

3. The rule set $\mathcal{R}_{S 4}$ is principal-cut closed, since $\operatorname{cut}\left(R_{4 \square}, R_{T \square}, \square p\right)=R_{\text {id }}$.

Since in the presence of the rules for (intuitionistic) propositional logic it is possible to re-construct the cut formula from the premisses of the cut between two rules, saturating a rule set under cuts between rules does not change the set of derivable sequents:

Lemma 9. If $\mathcal{R}$ is a set of rules and $R$ is a cut between two rules from $\mathcal{R}$, then $r_{\mathrm{G}[i] \mathrm{CutCon} \mathcal{R} R}^{[i]}$ $\Gamma \Rightarrow \Delta$ iff $\vdash_{\mathrm{G}[i] \mathrm{CutCon \mathcal {R }}}^{[\mathrm{R}} \Gamma \Rightarrow \Delta$.

Cuts between rules provide us with a means of eliminating cuts on principal formulae of two rules by replacing the cut with an instance of the cut between the two rules and a number of cuts on formulae of lower complexity. Moreover, Lemma 9 guarantees that we may simply add missing cuts to a rule set without jeopardising soundness. While this is enough for axioms without nested modalities [10], in the more general setting with context restrictions we need additional criteria for cuts involving context formulae:

Definition 10. Two restrictions $C_{1}=\left\langle F_{1}, F_{2}\right\rangle, C_{2}=\left\langle G_{1}, G_{2}\right\rangle$ overlap if there are formulae $A_{1} \in F_{2}, A_{2} \in G_{1}$ and substitutions $\sigma_{1}, \sigma_{2}$ with $A_{1} \sigma_{1}=A_{2} \sigma_{2}$. A rule set $\mathcal{R}$ is

1. context-cut closed if whenever $R_{0}, R_{1} \in \mathcal{R}$ and there are context restrictions $C_{0}$ of $R_{0}$ and $C_{1}$ of $R_{1}$ which overlap, then there is $i \in\{0,1\}$ such that all context restrictions of $R_{i}$ which overlap $C_{1-i}$ and the principal formulae of $R_{i}$ satisfy $C_{1-i}$.

2. mixed-cut closed if whenever $R, Q \in \mathcal{R}$ and a principal formula $A$ of $R$ satisfies a context restriction of $Q$, then all context restrictions of $R$ and all principal formulae of $R$ except for $A$ satisfy all those context restrictions of $Q$ satisfied by $A$.

Intuitively, these conditions allow pushing cuts involving context formulae into the premisses of one of the rules and eliminating them by induction on the cut level.

Example 11. 1. The rule sets $G[i]$ are context- and mixed-cut closed because all the rules involve only the restriction $C_{i d}$ or its asymmetric version $C_{i d}^{i}:=\langle\{p\}, \emptyset\rangle$. Hence every restriction is satisfied by every principal formula and every other restriction.

2. The rule set $\mathcal{R}_{K}$ is trivially context- and mixed-cut closed.

3. The rule set $\mathcal{R}_{S 4}$ is mixed-cut closed, since the restriction $C_{4 \square}$ satisfies $C_{i d}$. Since the principal formula of $R_{4 \square}$ also satisfies $C_{i d}$, the set is furthermore context-cut closed.

Since in general rules are not invertible and we need to take care of Contraction we will follow Gentzen's original strategy [8] when proving cut elimination and eliminate multicuts $\frac{\Gamma \Rightarrow \Delta, A^{n} \quad A^{m}, \Sigma \Rightarrow \Pi}{\Gamma, \Sigma \Rightarrow \Delta, \Pi}$ instead of cuts. Thus we also need to deal with multiple principal occurrences of the same formula. We do this by elevating contraction to the level of derivation rules and considering rule sets closed under this operation.

Definition 12 ([11]). If $\mathcal{P}$ is a set of premisses with restrictions and $\boldsymbol{p}=\left(p_{1}, \ldots, p_{n}\right)$ and $\boldsymbol{q}=\left(q_{1}, \ldots, q_{n}\right)$ are $n$-tuples of variables, then $\mathcal{P}[\boldsymbol{q} \leftarrow \boldsymbol{p}]$ is the result of replacing every occurrence of $q_{i}$ in a sequent occurring in a premiss in $\mathcal{P}$ by $p_{i}$ for all $i=1, \ldots, n$ 
and contracting duplicate instances of $p_{1}, \ldots, p_{n}$. Let $R=(\mathcal{P} ; \Gamma, \nabla \boldsymbol{p}, \nabla \boldsymbol{q} \Rightarrow \Delta)$ be a rule. The left contraction of $R$ on $\nabla \boldsymbol{p}$ and $\vee \boldsymbol{q}$ is the rule $\operatorname{ConL}(R, \nabla \boldsymbol{p}, \nabla \boldsymbol{q})=(\mathcal{P}[\boldsymbol{q} \leftarrow$ $\boldsymbol{p}] ; \Gamma, \nabla \boldsymbol{p} \Rightarrow \Delta)$. The right contraction $\operatorname{ConR}(R, \nabla \boldsymbol{p}, \nabla \boldsymbol{q})$ is defined dually. A rule set $\mathcal{R}$ is contraction closed if for every rule $R \in \mathcal{R}$ instances of the rules $\operatorname{ConL}(R, \nabla \boldsymbol{p}, \nabla \boldsymbol{q})$ and $\operatorname{ConR}(R, \nabla \boldsymbol{p}, \nabla \boldsymbol{q})$ can be simulated by applications of Weakening and Contraction, followed by at most one application of a rule $R^{\prime} \in \mathcal{R}$ and Weakening. A set of rules is saturated if it is contraction, principal-cut, context-cut, and mixed-cut closed.

Example 13. 1. The rules of $G[i]$ and $\mathcal{R}_{S 4}$ are trivially contraction closed.

2. $\mathcal{R}_{K}$ is contraction closed because $\operatorname{ConL}\left(R_{K_{n}}, \square p_{n-1}, \square p_{n}\right)=R_{K_{n-1}} \in \mathcal{R}_{K}$. Thus each of $\mathrm{G}[i], \mathcal{R}_{K}, \mathcal{R}_{S 4}$ are saturated.

Theorem 14 (Cut Elimination). For every saturated set $\mathcal{R}$ of rules and (asymmetric) sequent $\Gamma \Rightarrow \Delta$ we have $\vdash_{\mathcal{R C o n}}^{[i]} \Gamma \Rightarrow \Delta$ whenever $r_{\mathcal{R C o n C u t}}^{[i]} \Gamma \Rightarrow \Delta$.

While saturated rule sets allow for cut elimination, we are also interested in decision procedures via backwards proof search. For this we also need admissibility of Contraction. While contraction closure of the rule set takes care of contractions of two principal formulae of a rule, for contractions of principal and context formulae we use the standard method of copying the relevant principal formulae into the premisses. This might seem a bit coarse but again is necessary because in general the rules are not invertible.

Definition 15. For a rule $R=(\mathcal{P} ; \Sigma \Rightarrow \Pi)$ a modified instance

$$
\frac{\left\{(\Gamma, \Sigma \sigma) \uparrow_{F_{1}}, \Theta \sigma \Rightarrow(\Delta, \Pi \sigma) \uparrow_{F_{2}}, \Upsilon \sigma \mid\left(\Theta \Rightarrow \Upsilon ;\left\langle F_{1}, F_{2}\right\rangle\right) \in \mathcal{P}\right\}}{\Gamma, \Sigma \sigma \Rightarrow \Delta, \Pi \sigma}
$$

of $R$ is given by a substitution $\sigma: \mathcal{V} \rightarrow \mathcal{F}$ and a context $\Gamma \Rightarrow \Delta \in \mathcal{S}(\mathcal{F})$. We write $\vdash_{\mathcal{R}^{*}}$ for derivability using modified instances instead of instances of rules in $\mathcal{R}$.

Theorem 16 (Admissibility of Contraction). For every set $\mathcal{R}$ of rules and (asymmetric) sequent $\Gamma \Rightarrow \Delta$ we have $\vdash_{\mathcal{R C O n}}^{[i]} \Gamma \Rightarrow \Delta$ iff $\vdash_{\mathcal{R}^{*} \operatorname{Con}}^{[i]} \Gamma \Rightarrow \Delta$ iff $\vdash_{\mathcal{R}^{*}}^{[i]} \Gamma \Rightarrow \Delta$.

If the rule set is furthermore tractable in the sense that given a sequent the rules with this sequent as conclusion have codes of size polynomial in the size of the sequent, which can be recognised in space polynomial in the size of the sequent, and given the code of a rule its premisses can be recognised in space polynomial in the code, we get a generic complexity bound for deciding derivability using modified instances. Due to the more general rule format this bound is slightly higher than the PsPACE bound in [10]. Whether this can be improved in general is subject of ongoing work.

Theorem 17. For a saturated and tractable set $\mathcal{R}$ of rules, derivability in $\mathcal{R}^{*}$ is decidable in EXPTIME.

\subsection{Construction of Cut-Free Sequent Systems from Axioms}

With Theorems 14 and 16 we have presented a general criterion for a sequent system with context restrictions to admit both cut and contraction. Of course now we need to 
construct sequent systems satisfying this criterion. As in the case without context restrictions [10] these results suggest constructing saturated rule sets by saturation: starting with a set of rules with context restrictions simply add missing cuts and contractions until no more new rules are found. In the presence of context restrictions, however, we then need to check that the resulting rule set is also context-cut closed and mixed-cut closed. The following well-known example shows that this need not be the case.

Example 18. The rule set $\mathcal{R}_{S 5}:=\left\{\left(\left\{\left(p \Rightarrow ; C_{i d}\right)\right\} ; \square p \Rightarrow\right),\left(\left\{\left(\Rightarrow p ; C_{5 \square}\right)\right\} ; \Rightarrow \square p\right)\right\}$ with $C_{5 \square}:=\langle\{\square p\},\{\square p\}\rangle$ is contraction closed and principal- and context-cut closed. It is not mixed-cut closed, since the occurrence of the principal formula $\square p$ of $R_{T \square}$ satisfies the restriction $C_{5 \square}$ of $R_{5 \square}$, but the restriction $C_{i d}$ does not.

The method of constructing cut-free rule sets by saturation works reasonably well if we start with a set of sequent rules, but often the modal logics of interest are given in a Hilbert-style system by a set of axioms. Thus the first step in constructing a cut free sequent system from such axioms is to translate the axioms into sequent rules. While this can alway be done if the axioms are non-nested, i.e. without nested modalities, and the underlying propositional logic is classical, in the general case we need to be more careful. The notion of cuts between rules will be a useful tool in this step as well.

We assume that the underlying propositional logic is classical or intuitionistic. In a first step we extend the method for converting non-nested axioms from [10] to the asymmetric setting using notions from [6]. The main idea is to first treat the modal subformulae in a non-nested axiom like propositional variables, use invertibility of the underlying rule set to break the axiom into a finite number of sequents, and then resolve propositional logic under the modalities by introducing new variables and premisses stating that these variables are equivalent to the original formulae. Finally, these premisses are again broken up using invertibility of the underlying rules. To identify the axioms which can be broken up we loosely follow the idea of the substructural hierarchy from [6] and consider the notions of left resolvable and right resolvable formulae. Intuitively, if a right resolvable formula occurs positively in a sequent, its main (boolean) connective can be broken up. We introduce these notions in a generic form which allows treating classical and intuitionistic logics in the same framework. Also this shows that they are easily adapted to other logics such as minimal or distributive logic.

Definition 19. The sets $\mathcal{F}_{r}$ of right resolvable formulae and $\mathcal{F}_{\ell}$ of left resolvable formulae and their intuitionistic versions $\mathcal{F}_{r}{ }^{i}$ and $\mathcal{F}_{\ell}{ }^{i}$ are defined recursively by

1. if $p \in \mathcal{V}$ then $p \in \mathcal{F}_{r}^{[i]}$ and $p \in \mathcal{F}_{\ell}{ }^{[i]}$;

2. $\perp \in \mathcal{F}_{r}{ }^{[i]}$ and $\perp \in \mathcal{F}_{\ell}{ }^{[i]}$;

3. if $A_{1}, A_{2} \in \mathcal{F}_{r}^{[i]}$ then $A_{1} \wedge A_{2} \in \mathcal{F}_{r}^{[i]}$ and $A_{1} \vee A_{2} \in \mathcal{F}_{r}$;

4. if $A_{1}, A_{2} \in \mathcal{F}_{\ell}^{[i]}$ then $A_{1} \wedge A_{2} \in \mathcal{F}_{\ell}^{[i]}$ and $A_{1} \vee A_{2} \in \mathcal{F}_{\ell}^{[i]}$;

5. if $A_{1} \in \mathcal{F}_{\ell}^{[i]}$ and $A_{2} \in \mathcal{F}_{r}^{[i]}$ then $A_{1} \rightarrow A_{2} \in \mathcal{F}_{r}^{[i]}$;

6. if $A_{1} \in \mathcal{F}_{r}$ and $A_{2} \in \mathcal{F}_{\ell}$ then $A_{1} \rightarrow A_{2} \in \mathcal{F}_{\ell}$

where again we write $\mathcal{F}_{r}^{[i]}$ if a clause applies both to $\mathcal{F}_{r}$ and $\mathcal{F}_{r}{ }^{i}$.

Example 20. The formula $p \wedge((p \vee q) \rightarrow r)$ is intuitionistically right resolvable. Both $p \vee q$ and $(p \rightarrow q) \rightarrow \perp$ are classically right resolvable, but not intuitionistically. 
Since the premisses of the right (left) rule for the main connective of a right (left) resolvable formula can be derived from its conclusion in $\mathrm{G}[i]$ Cut, we may decompose an axiom $\overline{\Rightarrow A}$ for $A \in \mathcal{F}_{r}$ into a number of sequents over $\mathcal{V}$, similar to computing the regular normal form [15] of a formula:

Lemma 21. Let $\Gamma \subseteq \mathcal{F}_{\ell}^{[i]}$ and $\Delta \subseteq \mathcal{F}_{r}^{[i]}$. Then there are unique sequents $\Gamma_{i} \Rightarrow \Delta_{i} \in$ $\mathcal{S}(\mathcal{V})$ such that the axiom $\overline{\Gamma \Rightarrow \Delta}$ is equivalent in $\mathrm{G}[i]$ CutCon to $\overline{\Gamma_{1} \Rightarrow \Delta_{1}}, \ldots, \overline{\Gamma_{n} \Rightarrow \Delta_{n}}$.

This Lemma is used to break modal axioms into sequents by treating the modalised subformulae as variables. Then the propositional variables are moved into the premisses:

Lemma 22. For $\Gamma \Rightarrow \Delta \in \mathcal{S}(\mathcal{V})$ and $\Sigma \Rightarrow \Pi \in \mathcal{S}(\Lambda(\mathcal{V}))$ the axiom $\overline{\Gamma, \Sigma \Rightarrow \Delta, \Pi}$ is equivalent in $\mathrm{G}[i]$ CutCon to the rule $\left(\left\{\left(\Rightarrow p ; C_{i d}^{[i]}\right) \mid p \in \Gamma\right\} \cup\left\{\left(p \Rightarrow ; C_{i d}\right) \mid p \in\right.\right.$ $\Delta\} ; \Sigma \Rightarrow \Pi)$.

In the case of axioms with modal nesting depth one we now eliminate propositional logic under the modalities by adding new variables for the immediate subformulae and new premisses stating that the variables are equivalent to the original subformulae. Unfortunately the result of this operation is not necessarily a rule in our sense, since the sequents occurring in the premisses do not only include variables. Fortunately, if the modality in question is monotone or antitone (see below), we can cut this "rule" with the monotonicity rule (or its antitone counterpart) to eliminate one of the new premisses. We call the newly introduced premisses the premisses for the subformula $A$ and say that the premisses for a subformula can be resolved if there are equivalent premisses consisting of sequents over variables. The following Definition and Lemma give criteria on when the premisses can be resolved.

Definition 23. Let $\mathcal{R}$ be a set of rules, $\boldsymbol{p}=p_{1}, \ldots, p_{n}$ and $\boldsymbol{q}=q_{1}, \ldots, q_{n}$. For $k \leq n$ a $n$-ary modality $\nabla \boldsymbol{p}$ is monotone in the $k$-th argument if the rule $R_{\text {mon }_{k}}=\left(\left\{\left(p_{k} \Rightarrow\right.\right.\right.$ $\left.\left.\left.q_{k} ; C_{\emptyset}\right)\right\} \cup\left\{\left(p_{\ell} \Rightarrow q_{\ell} ; C_{\emptyset}\right) \mid k \neq \ell \leq n\right\} \cup\left\{\left(q_{\ell} \Rightarrow p_{\ell} ; C_{\emptyset}\right) \mid k \neq \ell \leq n\right\} ; \nabla \boldsymbol{p} \Rightarrow \nabla \boldsymbol{q}\right)$ is in $\mathcal{R}$. It is antitone in the $k$-th argument if the rule $R_{a n t_{k}}$ with premiss $\left(q_{k} \Rightarrow p_{k} ; C_{\emptyset}\right)$ instead of $\left(p_{k} \Rightarrow q_{k} ; \mathcal{C}_{\emptyset}\right)$ is in $\mathcal{R}$.

Lemma 24. Let $\mathcal{R}$ be a rule set. Then for a sequent $\Gamma \Rightarrow \Delta, \nabla\left(\ldots, A_{k}, \ldots\right)$ the premisses for $A_{k}$ can be resolved if: $A_{k} \in \mathcal{F}_{\ell}^{[i]}$ and $\triangleright$ is monotone in the $k$-th argument; or $A_{k} \in \mathcal{F}_{r}^{[i]}$ and $\triangleright$ is antitone in the $k$-th argument; or $A_{k} \in \mathcal{F}_{\ell}^{[i]} \cap \mathcal{F}_{r}^{[i]}$. For a sequent $\odot\left(\ldots, A_{k}, \ldots\right), \Gamma \Rightarrow \Delta$ we have the analogous result with $\mathcal{F}_{\ell}^{[i]}$ and $\mathcal{F}_{r}^{[i]}$ exchanged.

The previous Lemmata yield general criteria as to which axioms are translatable into rules. For the sake of brevity we only state the result for unary monotone modalities; The generalisations to non-monotone modalities and higher arities are straightforward.

Definition 25. For $A \in \mathcal{F}_{r}{ }^{[i]}$ and $p \in \mathcal{V}$ we say that $p$ is positive (resp. negative) in $A$, if it occurs positively (resp. negatively) in a sequent of the decomposition of the sequent $\Rightarrow A$ according to Lemma 21 .

Theorem 26. Let $A$ be a propositional formula with variables $p_{1}, \ldots, p_{n}, q_{1}, \ldots, q_{m}$, and for $i=1, \ldots, m$ let the modality $\nabla_{i}$ be unary monotone and $A_{i}$ a propositional 
formula with variables in $p_{1}, \ldots, p_{n}$ such that: $q_{i}$ is only positive in $A$ and $A_{i} \in \mathcal{F}_{\ell}^{[i]}$; or $q_{i}$ is only negative in $A$ and $A_{i} \in \mathcal{F}_{r}^{[i]}$; or $A \in \mathcal{F}_{\ell}^{[i]} \cap \mathcal{F}_{r}{ }^{[i]}$. Then there is a rule which is equivalent in $\mathrm{G}[i]$ CutCon to the axiom $\overline{\Rightarrow A \sigma}$ where $\sigma\left(q_{i}\right)=\vee_{i}\left(A_{i}\right)$ and $\sigma\left(p_{i}\right)=p_{i}$.

Remark 27. Since for classical propositional logic all propositional formulae are both right and left resolvable, the previous Theorem yields the translation result for nonnested axioms from [10] as a Corollary.

For axioms with nested modalities we may sometimes use a similar procedure if a modalised formula occurs both on the top level of the axiom and under a modality. The idea is to introduce a fresh variable for this formula and apply the methods above to resolve propositional logic under the modalities, but without moving the top level occurrences of the variable into the premisses with Lemma22 If now the occurrences of this variable in the premisses and the conclusion are all negative (resp. positive), we may it replace it again with the original formula. Since this formula now occurs both in the premisses and the conclusion this often gives rise to a context restriction. We will illustrate this method using examples in the next section.

\section{Applications}

Example 28 (Constructive $K$ ). Constructive modal logic $K$ from [4]13] is based on intuitionistic propositional logic and has rules $\operatorname{Reg}_{\square}=\left(\left\{\left(p \Rightarrow q ; C_{\emptyset}\right)\right\} ; \square p \Rightarrow \square q\right)$ and $\operatorname{Reg}_{\diamond}=\left(\left\{\left(p \Rightarrow q ; C_{\emptyset}\right)\right\} ; \diamond p \Rightarrow \diamond q\right)$ and axioms (FS1) $\square \mathrm{T},(F S 2)(\square(p \wedge q) \rightarrow$ $(\square p \wedge \square q)) \wedge((\square p \wedge \square q) \rightarrow \square(p \wedge q))$ and $(F S 6) \diamond(p \rightarrow q) \rightarrow(\square p \rightarrow \diamond q)$. Since the propositional part is intuitionistic we base our treatment on the asymmetric setting. The rules $R e g_{\square}$ and $R e g_{\diamond}$ ensure that both modalities $\square$ and $\diamond$ are monotone in the sense of Definition 23. Treating modalised subformulae as variables and using Lemma 21 and the fact that $A \rightarrow B$ is intuitionistically left resolvable to break up axiom (FS6) first yields the axiom $\overline{\diamond(p \rightarrow q), \square p \Rightarrow \diamond q}$. Now introducing a new variable $r_{p \rightarrow q}$ and premisses for subformula $p \rightarrow q$ yields

$$
\frac{r_{p \rightarrow q} \Rightarrow p \rightarrow q \quad p \rightarrow q \Rightarrow r_{p \rightarrow q}}{\diamond r_{p \rightarrow q}, \square p \Rightarrow \diamond q} .
$$

But now a cut with rule $R e g_{\diamond}$ on $\diamond r_{p \rightarrow q}$ and resolving the remaining premiss gives the rule $\frac{s, p \Rightarrow q}{\diamond s, \square p \Rightarrow \diamond q} R_{F S 6}$. The analogous treatment for axioms (FS2) and (FS1) gives the well-known rules $R_{F S 2}:=\left(\left\{\left(p, q \Rightarrow r ; C_{\emptyset}\right)\right\} ; \square p, \square q \Rightarrow \square r\right)$ and $R_{F S 1}:=(\{(\Rightarrow$ $\left.\left.\left.p ; C_{\emptyset}\right)\right\} ; \Rightarrow \square p\right)$. Now saturating the rule set under cuts yields the rule set

$$
\mathcal{R}_{C K}:=\left\{\frac{p_{1}, \ldots, p_{n} \Rightarrow q}{\Gamma, \square p_{1}, \ldots, \square p_{n} \Rightarrow \square q} R_{C K_{n}} \mid n \geq 0\right\} \cup\left\{\frac{p_{1}, \ldots, p_{n}, q \Rightarrow r}{\Gamma, \square p_{1}, \ldots, \square p_{n}, \diamond q \Rightarrow \diamond r} \mid n \geq 0\right\} .
$$

Of course this rule set is not new [4]. The point here is that we constructed it in a purely syntactical way from the axioms of the Hilbert-system.

To illustrate the use of Lemma 22 let us add the $T$-axioms (Ta) $\square p \rightarrow p$ and $(T \diamond) p \rightarrow \diamond p$. Again, the axioms are first broken up into $\overline{\square p \Rightarrow p}$ and $\overline{p \Rightarrow \diamond p}$. Then 
they are transformed into equivalent rules $R_{T \square}=\left(\left\{\left(p \Rightarrow ; C_{i d}\right)\right\} ; \square p \Rightarrow\right)$ and $R_{T \diamond}=$ $\left(\left\{\left(\Rightarrow p ; C_{i d}^{i}\right)\right\} ; \Rightarrow \diamond p\right)$. Now saturation under cuts would yield the additional rules $\left(\left\{\left(p_{1}, \ldots, p_{n} \Rightarrow ; C_{i d}\right)\right\} ; \square p_{1}, \ldots, \square p_{n} \Rightarrow\right)$ and $\left(\left\{\left(p_{1}, \ldots, p_{n} \Rightarrow r ; C_{i d}^{i}\right)\right\} ; \square p_{1}, \ldots, \square p_{n} \Rightarrow\right.$ $\diamond r)$ for $n \geq 0$, but these are simulated by repeated applications of $R_{T \square}$ and $R_{T \diamond}$. Thus it is easy to see that the rule sets $\mathcal{R}_{C K}$ and $\mathcal{R}_{C K} \cup\left\{R_{T \square}, R_{T \diamond}\right\}$ are saturated.

Note that these constructions do not give rise to context restrictions apart from $\mathcal{C}_{\emptyset}$ and $C_{i d}^{[i]}$. For this we need to consider axioms with nested modalities. Unfortunately, if we are dealing with nested modalities the translation becomes more involved and much less automatic. Nonetheless, as mentioned before in some cases we can still use the method of cutting rules on principal formulae to construct rules with context restrictions. The main idea is to make use of formulae occurring both under a modality and on the top level of the sequent and to construct a context restriction out of this formula.

Example 29 (Constructive S4). Constructive modal logic CS4 from [17|2] contains

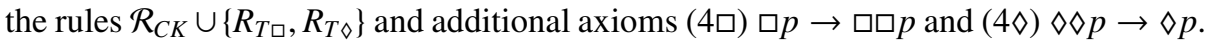
We make use of the fact that in these we have the same modalised formula occurring on the top level and under a modality as follows: Take axiom (4ロ) and in a first step replace the occurrence of $\square p$ under the modality by a fresh variable $q$. The resulting axiom $\square p \rightarrow \square q$ is broken up into the sequent $\square p \Rightarrow \square q$. Then adding the premisses for $q$ we get $\frac{\square p \Rightarrow q \quad q \Rightarrow \square p}{\square p \Rightarrow \square q}$ and a cut with the monotonicity rule $\operatorname{Reg}_{\square}$ yields $\frac{\square p \Rightarrow q}{\square p \Rightarrow \square q}$. Now computing principal cuts of a number of instances of this rule with rule $R_{C K_{n}}$ yields

$$
\frac{\square p_{1}, \ldots, \square p_{m}, q_{1}, \ldots, q_{k} \Rightarrow r}{\Gamma, \square p_{1}, \ldots, \square p_{m}, \square q_{1}, \ldots, \square q_{k} \Rightarrow \square r} \text {. }
$$

But since the $\square p_{i}$ occur both in conclusion and premiss of the rule, this is exactly the rule $R_{4 \square}=\left(\left\{\left(q_{1}, \ldots, q_{k} \Rightarrow r ; C_{4 \square}\right)\right\} ; \square q_{1}, \ldots, \square q_{k} \Rightarrow \square r\right)$. Moreover, the rule $\frac{\square p \Rightarrow q}{\square p \Rightarrow \square q}$ is sound by the methods of the last section, and since $R_{4 \square}$ was constructed from this rule and $R_{C K_{n}}$ by means of principal cuts, Lemma 9 ensures that it is sound as well. A similar process for axiom $(4 \diamond)$ yields the rules $R_{4 \diamond}:=\left(\left\{\left(p_{1}, \ldots, p_{n}, q \Rightarrow\right.\right.\right.$ $\left.\left.\left.C_{4 \diamond}\right)\right\} ; \square p_{1}, \ldots, \square p_{n}, \diamond q \Rightarrow\right)$ with context restriction $C_{4 \diamond}=\langle\emptyset,\{\diamond p\}\rangle$. Now adding the missing principal cuts again yields a rule set which is principal-cut closed. It is trivially context-cut closed, and easily checked to be mixed-cut and contraction closed and therefore saturated. Again, the rules are not new, but we constructed them in a purely syntactical way, and their soundness and completeness is guaranteed by construction.

This method can also be applied if the subformula occurs under a modality more than once, or if it is more complex. In the latter case in general this gives rise to more complex context restrictions. The following example shows how sometimes more complex restrictions can be simplified and how context restrictions $C_{i d}^{[i]}$ may arise other than as a consequence of Lemma22.

Example 30 (Access Control Logic $C D D$ ). Access control logic $C D D$ from [1] is based on intuitionistic propositional logic and has indexed normal (and thus monotone) modalities $\bigcirc_{k} A$ which are interpreted as principal $k$ says $A$. For this example we consider the axiomatisation with the axioms [unit] $p \rightarrow \bigcirc_{k} p$ and [GHO] $\bigcirc_{k}$ $\left(p \rightarrow \bigcirc_{k} q\right) \rightarrow\left(p \rightarrow \bigcirc_{k} q\right)$ (see [1]). The first axiom straightforwardly translates into 
the rule $R_{\text {[unit] }}=\left(\left\{\left(\Rightarrow p ; C_{i d}^{i}\right)\right\} ; \Rightarrow \bigcirc_{k} p\right)$. For the latter axiom we first introduce a variable $r$ for the formula $\left(p \rightarrow \bigcirc_{k} q\right.$ ) under the modality and apply the methods above to obtain

$$
\frac{r \Rightarrow p \rightarrow \bigcirc_{k} q}{\Gamma, \bigcirc_{k} r \Rightarrow p \rightarrow \bigcirc_{k} q}
$$

But now instead of turning this into a rule with context restriction $\left\langle\emptyset,\left\{p \rightarrow \bigcirc_{k} q\right\}\right\rangle$ we break up the boolean part to arrive at $\frac{r, p \Rightarrow \bigcirc_{k} q}{\Gamma, \bigcirc_{k} r, p \Rightarrow \bigcirc_{k} q}$. Since disjunctions are intuitionistically left resolvable the variable $p$ can be taken to be the context on the left hand side, and this is equivalent to the rule $R_{[\mathrm{GHO}]}=\left(\left\{\left(r \Rightarrow ; C_{c d d, k}\right)\right\} ; \bigcirc_{k} r \Rightarrow\right)$ with restriction $C_{c d d, k}:=\left\langle\{p\},\left\{\bigcirc_{k} p\right\}\right\rangle$. Since the rules $R_{K_{n}}$ for $\bigcirc_{k}$ are simulated by applications of $R_{\text {[unit] }}$ and $R_{[\mathrm{GHO}]}$, this yields the rule set $\mathcal{R}_{C D D}:=\left\{R_{\text {[unit] }}, R_{[\mathrm{GHO}]}\right\}$ which again is easily seen to be saturated and thus have cut elimination. Of course again this rule set is not new (see e.g. [7/5]) and there are other ways to construct it, but it nicely illustrates how context restrictions arise.

Example 31 (Conditional Logic with Absoluteness). As a final example let us construct a cut-free set of rules with context restrictions for a logic based on classical propositional logic, namely for Lewis' conditional logic $\mathbb{V} \mathbb{A}$ from [12]. The language for this logic contains the binary modality $\leqslant$ called comparative plausibility operator with the intuitive reading " $A$ is at least as plausible as $B$ " for $A \leqslant B$. The logic $\mathbb{V} \mathbb{A}$ is given as an axiomatic extension of the logic $\mathbb{V}$, where the latter is characterised by non-nested axioms only and does not necessitate the use of rules with context restrictions. For this reason we concentrate on the new axioms and make use of the rules $\mathcal{R}_{\mathbb{V}}=\left\{R_{n, m} \mid n \geq 1, m \geq 0\right\}$ from [11] for the logic $\mathbb{V}$, where rule $R_{n, m}$ in our notation is given as $\left(\left\{\left(s_{k} \Rightarrow r_{1}, \ldots, r_{n}, q_{1}, \ldots, q_{m} ; C_{\emptyset}\right) \mid k \leq n\right\} \cup\left\{\left(p_{k} \Rightarrow\right.\right.\right.$ $\left.\left.\left.r_{1}, \ldots, r_{n}, q_{1}, \ldots, q_{k-1} ; C_{\emptyset}\right) \mid k \leq m\right\} ; p_{1} \leqslant q_{1}, \ldots, p_{m} \leqslant q_{m} \Rightarrow r_{1} \leqslant s_{1}, \ldots, r_{n} \leqslant s_{n}\right)$.

For $\mathbb{V} \mathbb{A}$ we need to add the two absoluteness axioms $(p \leqslant q) \rightarrow(\perp \leqslant \neg(p \leqslant q))$ and $\neg(p \leqslant q) \rightarrow(\perp \leqslant(p \leqslant q))$. We use the fact that the formula $(p \leqslant q)$ occurs both on the top level and under a modality, and in a first step using monotonicity of $\leqslant$ in the second argument convert the two axioms into $\frac{p \Rightarrow q \Rightarrow r \leqslant s}{\Rightarrow p \leqslant q, r \leqslant s}$ and $\frac{p \Rightarrow r \leqslant s \Rightarrow p \leqslant q}{r \leqslant s, q \Rightarrow}$. Now computing a principal cut between the first of these rules and a rule $R_{n, m}$ effectively replaces one negative principal formula of $R_{n, m}$ with a positive contextual formula $r \leqslant$ $s$. Repeating this process we get arbitrarily many positive context formulae $r_{k} \leqslant s_{k}$ and thus arrive at the context restriction $\langle\emptyset,\{r \leqslant s\}\rangle$. Similarly, principal cuts with the second rule replace negative principal formulae of $R_{n, m}$ with negative contextual formulae $r \leqslant s$, yielding the context restriction $C_{\mathbb{V A}}:=\langle\{r \leqslant s\},\{r \leqslant s\}\rangle$. As usual, since all the cuts involved were cuts on principal formulae, Lemma 9 guarantees soundness of the resulting rule set. Setting $\mathcal{R}_{\mathbb{V A}}=\left\{R_{n, m}^{\prime} \mid n \geq 1, m \geq 0\right\}$ with $R_{n, m}^{\prime}$ given as $\left(\left\{\left(s_{k} \Rightarrow r_{1}, \ldots, r_{n}, q_{1}, \ldots, q_{m} ; C_{\mathbb{V} A}\right) \mid k \leq n\right\} \cup\left\{\left(p_{k} \Rightarrow r_{1}, \ldots, r_{n}, q_{1}, \ldots, q_{k-1} ; C_{\mathbb{V A}}\right) \mid\right.\right.$ $\left.k \leq m\} ; p_{1} \leqslant q_{1}, \ldots, p_{m} \leqslant q_{m} \Rightarrow r_{1} \leqslant s_{1}, \ldots, r_{n} \leqslant s_{n}\right)$ thus gives a sound and complete rule set for $\mathbb{V} \mathbb{A}$, which is easily checked to be saturated and thus cut-free. As far as we are aware this rule set is new. This yields the following Theorem. 
Theorem 32. The rule set $\mathrm{G} \mathcal{R}_{\mathbb{V} A}$ ConCut is sound and complete for $\mathbb{V} \mathbb{A}$. Moreover, it is saturated and therefore has cut elimination. Since $\mathcal{R}_{\mathbb{V}}$ is tractable, derivability in this system can be checked in EXPTIME.

\section{Conclusion}

We presented a generic cut elimination result for symmetric and asymmetric sequent systems consisting of rules with context restrictions which are saturated, i.e. closed under cuts and contractions. This not only extends previous methods to modal axioms of nesting depth greater than one, but also to logics based on intuitionistic logic. Furthermore, we introduced techniques to translate axioms of a Hilbert style system into sequent rules. All the results and techniques are easily adapted to other base logics such as minimal or distributive logic. Examples included the reconstruction of already known sequent systems for constructive modal logics and the construction of an apparently new sequent system for Lewis' conditional logic $\mathbb{V} \mathbb{A}$ in the entrenchment language.

\section{References}

1. Abadi, M.: Variations in Access Control Logic. In: van der Meyden, R., van der Torre, L. (eds.) DEON 2008. LNCS (LNAI), vol. 5076, pp. 96-109. Springer, Heidelberg (2008)

2. Alechina, N., Mendler, M., de Paiva, V., Ritter, E.: Categorical and Kripke Semantics for Constructive S4 Modal Logic. In: Fribourg, L. (ed.) CSL 2001 and EACSL 2001. LNCS, vol. 2142, pp. 292-307. Springer, Heidelberg (2001)

3. Avron, A., Lahav, O.: Kripke Semantics for Basic Sequent Systems. In: Brünnler, K., Metcalfe, G. (eds.) TABLEAUX 2011. LNCS (LNAI), vol. 6793, pp. 43-57. Springer, Heidelberg (2011)

4. Bellin, G., de Paiva, V., Ritter, E.: Extended Curry-Howard correspondence for a basic contructive modal logic. In: Areces, C., de Rijke, M. (eds.) M4M-2. ILLC Amsterdam (2001)

5. Benton, P., Bierman, G., de Paiva, V.: Computational types from a logical perspective. J. Funct. Programming 8(2), 177-193 (1998)

6. Ciabattoni, A., Galatos, N., Terui, K.: From axioms to analytic rules in nonclassical logics. In: LICS 2008, pp. 229-240. IEEE Computer Society (2008)

7. Fairtlough, M., Mendler, M.: Propositional lax logic. Inform. and Comput. 137(1), 1-33 (1997)

8. Gentzen, G.: Untersuchungen über das logische Schließen. I. Math. Z. 39(2), 176-210 (1934)

9. Johansson, I.: Der Minimalkalkül, ein reduzierter intuitionistischer Formalismus. Compos. Math. 4, 119-136 (1937)

10. Lellmann, B., Pattinson, D.: Cut Elimination for Shallow Modal Logics. In: Brünnler, K., Metcalfe, G. (eds.) TABLEAUX 2011. LNCS, vol. 6793, pp. 211-225. Springer, Heidelberg (2011)

11. Lellmann, B., Pattinson, D.: Sequent Systems for Lewis' Conditional Logics. In: del Cerro, L.F., Herzig, A., Mengin, J. (eds.) JELIA 2012. LNCS, vol. 7519, pp. 320-332. Springer, Heidelberg (2012)

12. Lewis, D.: Counterfactuals. Blackwell (1973)

13. Mendler, M., Scheele, S.: Cut-free Gentzen calculus for multimodal CK. Inform. and Comput. 209, 1465-1490 (2011)

14. Negri, S.: Proof analysis in modal logic. J. Philos. Logic 34, 507-544 (2005) 
15. Negri, S., von Plato, J.: Structural proof theory. Cambridge University Press (2001)

16. Pattinson, D., Schröder, L.: Generic modal cut elimination applied to conditional logics. Log. Methods Comput. Sci. 7(1) (2011)

17. Pfenning, F., Davies, R.: A judgmental reconstruction of modal logic. Math. Structures Comput. Sci. 11(4), 511-540 (2001)

18. Troelstra, A.S., Schwichtenberg, H.: Basic Proof Theory, 2nd edn. Cambridge Tracts Theoret. Comput. Sci. Cambridge University Press (2000)

19. Wansing, H.: Sequent systems for modal logics. In: Gabbay, D.M., Guenthner, F. (eds.) Handbook of Philosophical Logic, vol. 8. Springer (2002) 